rev.relac.int.estrateg.segur.11(2):75-92,2016

\title{
LAS NECESIDADES MUNDIALES DE AGUA Y LA ANTÁRTIDA COMO RESERVA NATURAL: ¿PUEDE EXPLOTARSE EL AGUA ANTÁRTICA?*
}

\author{
Fernando Villamizar Lamus**
}

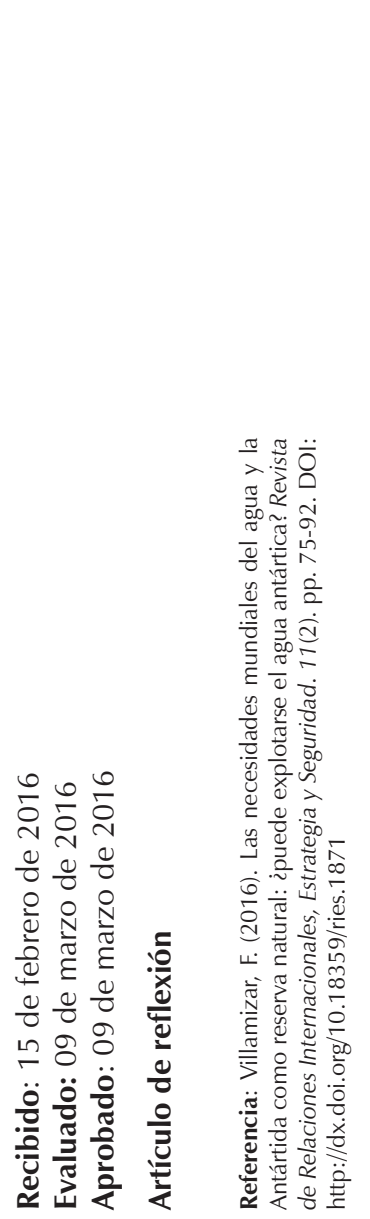

\section{RESUMEN}

La carencia de agua potable es cada vez más sensible para satisfacer las crecientes demandas mundiales. En ese escenario, la Antártida, una de las mayores reservas de agua, puede jugar un rol muy importante, especialmente por la creciente tendencia de creación de instrumentos internacionales e interpretaciones de los mismos para que los Estados garanticen el derecho al agua potable. El presente artículo tiene como objetivo analizar, mediante un método cualitativo, si jurídicamente sería posible explotar agua antártica, pese a las prohibiciones contenidas en el Protocolo de Madrid. Para ese fin, se estudiarán los instrumentos

\footnotetext{
Observatorio Regional de Paz y Seguridad (ORPAS), Universidad Bernardo O'Higgins (Chile). El artículo es resultado del Programa de Investigación e Intercambio Estudiantil y de Profesores "La Soberanía en Territorio Antártico" entre la Escuela de Derecho de la Universidad Bernardo O'Higgins (Chile) y la Facultad Teresa de Ávila de la Pontificia Universidad Católica Argentina (sede Paraná).

** Abogado, Universidad del Rosario. Magíster en Derecho de la empresa, Pontificia Universidad Católica de Chile. Doctor Summa Cum Laude en Ciencia Política y Sociología, Universidad Pontifica de Salamanca. Investigador del Observatorio Regional de Paz y Seguridad, Universidad Bernardo O'Higgings. Profesor de la Facultad de Derecho de la Universidad de los Andes (Chile). Correo electrónico: fvillamizar@miuandes.cl
} 
internacionales que pretenden obligar a los Estados a satisfacer las necesidades de agua potable a sus ciudadanos. A reglón seguido, se estudia la estructura del Sistema del Tratado Antártico y cómo la Antártida podría ser una solución para satisfacer las crecientes necesidades del recurso hídrico, particularmente por lo acordado en el Acta Final de la XI Reunión Consultiva Especial del Tratado Antártico, que puede ser la llave para la explotación de agua antártica, en forma de hielo, pero que los dilemas políticos que esa práctica conllevaría requieren de un consenso muy difícil de lograr, salvo que sea por situaciones excepcionales que lo permitan.

Palabras clave: Agua; Antártida; Conflicto; Minerales

\title{
THE WORLD NEEDS OF WATER AND THE ANTARCTICA AS A NATURAL RESERVATION: IT CAN BE EXPLOITED THE ANTARCTIC WATER
}

\begin{abstract}
Lack of clean water is increasingly sensitive to meet the growing global demands. In this scenario, Antarctica, one of the largest reserves of water, can play an important role, especially in the growing trend of developing international instruments and interpretations thereof for States to ensure the right to drinking water. This article aims to analyze, through a qualitative method, if legally it would be possible to exploit Antarctic water, despite the prohibitions contained in the Madrid Protocol. To that end, the international instruments that seek to oblige States to meet the needs of drinking water to its citizens will be explored. Immediately afterwards, studies the structure of the Antarctic Treaty System and how Antarctica might be a solution to meet the growing needs of water resources, particularly as agreed in the Final Act of the Eleventh Special Antarctic Treaty Consultative Meeting. It will be found that the Act can be the key to exploiting Antarctic water as ice, but that the political dilemmas that require practice entail a very difficult consensus to achieve, unless in exceptional circumstances permitted.
\end{abstract}

Key words: Antarctica, Conflict, Minerals, Water.

\section{AS NECESSIDADES MUNDIAIS DA ÁGUA E A ANTÁRTIDA COMO RESERVA NATURAL: SE PODERÁ EXPLORAR A ÁGUA ANTÁRCTICA?}

\section{RESUMO}

A falta de água potável é cada vez mais sensível para atender às crescentes demandas globais. Neste cenário, Antártida, uma das maiores reservas de 
água, podem desempenhar um papel importante, especialmente na tendência crescente de desenvolvimento de instrumentos internacionais e interpretações dos mesmos para os Estados para garantir o direito à água potável. Este artigo tem por objetivo analisar, por meio de um método qualitativo, se legalmente seria possível explorar águas da Antártida, apesar das proibições contidas no Protocolo de Madrid. Para o efeito, os instrumentos internacionais que visam obrigar os Estados para satisfazer as necessidades de água potável para os seus cidadãos serão exploradas. Imediatamente depois, estuda a estrutura do Sistema do Tratado Antártico e como Antártida pode ser uma solução para atender às necessidades crescentes de recursos hídricos, em particular, tal como pactuado na Acta Final da XI Reunião Consultiva Especial do Tratado Antártico, que poderá ser a chave para a exploração de água antártica, em forma de gelo, mas que os dilemas políticos que requerem essa prática implica um consenso muito difícil de conseguir, a não ser em circunstâncias excepcionalmente permitidas.

Palavras-chave: Água; Antartida; Conflito; Minerais

\section{Introducción}

A comienzos de diciembre de 2014 los titulares de algunos medios de comunicación daban cuenta de los esfuerzos de China para enviar más de 100 toneladas de agua a Maldivas, con el fin de paliar la crisis de agua que afectaba las islas (Xinhuanet, 2014). Aunque esta crisis se debió a un hecho puntual, el incendio de la única planta desalinizadora, es una muestra patente de las dificultades que poco a poco se presentan para suministrar el líquido vital a ingentes masas de población que la requieren en diferentes puntos del orbe terráqueo.

Si ya la situación de acceso al agua potable es difícil, esta se torna más preocupante al apreciar las prospecciones sobre la disponibilidad de recursos hídricos para finales de siglo. Diferentes zonas del mundo van a carecer del agua suficiente para satisfacer sus necesidades, entre estas Europa, el norte y el sur de África, y vastas zonas de América, entre otras (Diplomatie, Décembre - Janvier 2014). Este panorama se agrava aún más como consecuencia del cambio climático y por los daños ambientales que afectan ese recurso en todo el orbe (Harris, 2014), así como por la creciente demanda de recursos hídricos por parte de ciertas potencias emergentes, como India, China, Pakistán e Indonesia, entre otros, y por la de por sí alta demanda de potencias como Estados Unidos y Japón (Diplomatie, Décembre Janvier 2014).

En ese orden de ideas, la escasez de agua es un imperativo que puede alterar el orden mundial. De hecho, 
el informe de la Comunidad de Inteligencia de Estados Unidos de 2014 resalta que la falta de recursos como el agua puede generar conflictos internos e internacionales, así como inestabilidad en el gobierno (Office of the Director of National Intelligence, 2014) ${ }^{3}$. Desde luego, la falta de agua genera un serio ánimo por conquistarla para quienes no tienen el recurso y para quienes lo tienen, el esfuerzo por no compartirla, con lo cual los conflictos serán inevitables y con consecuencias funestas.

Ante las diferentes crisis que se han dado en varios lugares del mundo, algunos estatutos jurídicos e interpretaciones de los mismos apuntan a garantizar el acceso al agua potable como un derecho humano vital, porque es evidente que sin ese recurso es inviable cualquier posibilidad de vida. Así las cosas, la búsqueda de agua potable se convierte en una razón imprescindible para la vida en el planeta y el orden mundial, y la pregunta que surge es ien dónde se puede hallar el agua potable suficiente para evitar los efectos funestos de sequías e impedimentos para el acceso al agua?

Hay un continente que tiene los recursos necesarios para evitar dichos efectos y que podría ser la potencial salvación para las zonas afectadas por la ausencia o escasez de agua: la Antártida. En efecto, según algunas estimaciones mayoritariamente aceptadas, en la Antártida se encuentra el $90 \%$ del hielo del planeta y el $70 \%$ del agua potable del mundo (Ferrigno et al. 2009). Así las cosas, es evidente preguntarse: ipodría utilizarse el agua dulce de la Antártida para atender la creciente necesidad de agua del planeta? Este cuestionamiento es el problema de investigación que se pretende resolver, bajo la hipótesis de que hay posibilidades desde un punto de vista jurídico de explotar el hielo antártico, que podría convertirse en agua, pese a las disposiciones de prohibición de explotación de minerales contenidas en el artículo 7 del Protocolo al Tratado Antártico sobre Protección del Medio Ambiente, mejor conocido como Protocolo de Madrid, que impide cualquier actividad relacionada con los recursos minerales, salvo para fines de investigación científica.

En las líneas subsiguientes trataremos de dar una respuesta a esta pregunta, considerando algunas de las disposiciones e interpretaciones de estatutos internacionales, que elevan el derecho al acceso al agua como derecho humano fundamental, por 
lo cual sería imperativo explotar los recursos hídricos antárticos para salvar vidas, y las regulaciones contenidas en el Sistema del Tratado Antártico.

Para esos efectos, en primer lugar se analizarán los diversos estatutos internacionales que tratan el tema del agua, en segundo lugar se hará ídem respecto del régimen establecido en el Sistema del Tratado Antártico y se analizará el Acta Final de la XI Reunión Consultiva Especial del Tratado Antártico (en adelante Acta Final), documento por el cual se abre la ruta hacia la posibilidad jurídica de explotar agua antártica, siempre que sea en forma de hielo. Finalmente, se expondrán las respectivas conclusiones que emanarán de la comprobación de la hipótesis.

\section{Tratados y regulaciones interna- cionales por los cuales los Estados deben satisfacer las necesidades de agua de la población}

Aunque el hecho de que el agua es esencial para la vida y no se debiera requerir mayor proceso mental para llegar a ello, ha sido menester establecer esa necesidad vital en instrumentos internacionales para resguardar derechos y obligaciones que permitan el acceso a ese precioso líquido ${ }^{4}$. Entre los instrumentos inter-nacionales, por los cuales se ha interpretado ${ }^{5}$ que existe un derecho humano al agua está la Declaración Universal de Derechos Humanos, que en su artículo 25 dice: "Toda persona tiene derecho a un nivel de vida adecuado que le asegure, así como a su familia, la salud y el bienestar, y en especial la alimentación, el vestido, la vivienda".

También el Pacto Internacional de Derechos Económicos, Sociales y Culturales del 16 de diciembre de 1966, establece en los artículos 11.1: "Los Estados Partes en el presente Pacto reconocen el derecho de toda persona a un nivel de vida adecuado para sí y su familia, incluso alimentación, vestido y vivienda adecuados, y a una mejora continua de las condiciones de existencia"; y 12.1: "Los Estados Partes en el presente Pacto reconocen el derecho de toda persona al disfrute del más alto nivel posible de salud física y mental".

Junto con los instrumentos anteriores, la profesora chilena Silvia Bertazzo

Es menester aclarar que las nuevas tendencias interpretan que el acceso al agua y el saneamiento debieran ser tratados como dos derechos humanos distintos. En ese sentido puede consultarse la opinión de la relatora especial sobre el derecho humano al agua potable y al saneamiento, Catarina De Albuquerque (2012:27)

5 Sobre ese sentido de las interpretaciones puede consultarse, entre otros, a Martínez y Defelipe (2013), Motta (2010), Pinto et al., 2008), United Nations Economic Commission for Europe (2013), Winker (2012). 
(2015) hace un estudio pormenorizado de los diferentes instrumentos internacionales por los cuales se puede interpretar el derecho humano al acceso al agua potable, aunque no esté expresamente regulado con ese tenor literal. Entre los instrumentos más destacados se encuentran, según la mencionada profesora, la Convención sobre la eliminación de todas las formas de discriminación contra la mujer (1979), la Convención sobre los derechos del niño (1989), la Convención sobre los derechos de las personas con discapacidad (2006), el Protocolo Adicional a la Convención Americana sobre Derechos Humanos (1988), y de manera más concreta hay casos en los cuales existen disposiciones que aluden al derecho al agua en circunstancias muy específicas, como es el caso del Convenio de Ginebra relativo al trato debido a los prisioneros de guerra, del 12 de agosto de 1949, que en su artículo 26, párrafo 3, contempla que "se suministrará a los prisioneros de guerra suficiente agua potable".

Adicionalmente a los instrumentos anteriores y con el fin de hacer constar de manera expresa que el derecho al agua es un derecho humano y no depender de interpretaciones que hagan esa consideración, aunque sean consideradas "soft law" ${ }^{6}$, en noviembre de 2002, el Comité de Derechos Económicos, Sociales y Culturales adoptó la Observación General № 15 sobre el derecho al agua. El artículo I.1 establece que "El derecho humano al agua es indispensable para una vida humana digna". La Observación № 15 también define el derecho al agua como el derecho de cada uno a disponer de agua suficiente, saludable, aceptable, físicamente accesible y asequible para su uso personal y doméstico.

A su vez, el 28 de julio de 2010, a través de la Resolución 64/292, la Asamblea General de las Naciones Unidas reconoció explícitamente el derecho humano al agua $y$ al saneamiento, reafirmando que un agua potable limpia y el saneamiento son esenciales para la realización de todos los derechos humanos. La Resolución exhorta a los Estados y organizaciones internacionales a proporcionar recursos financieros, a propiciar la capacitación y la transferencia de tecnología para ayudar a los países, en particular a los países en desarrollo, a proporcionar un suministro de agua potable y saneamiento saludable, limpio, accesible y asequible para todos.

\footnotetext{
$6 \quad$ Este concepto se utiliza para indicar que el instrumento o disposición en cuestión no es en sí mismo una la ley, es decir, no tiene un carácter jurídicamente vinculante, pero su importancia dentro del marco general del desarrollo jurídico internacional es tal que requiere una atención especial (Shaw, 2011).
} 
La consecuencia que emana de estos textos para los Estados que son parte de la Organización de Naciones Unidas, en particular de la Resolución 64/292, según la experta Maude Barlow es que estos deben preparar un plan de acción para hacer posible el acceso al agua y al saneamiento, y reportarlo al Comité de Derechos Económicos, Sociales y Culturales de la ONU. Esto supone tres obligaciones para los Estados, por una parte la obligación de respetar los derechos al agua, incluso dándoselas a quienes no pueden pagar por esta; la obligación de proteger los derechos al agua de interferencias por parte de terceros, así como de la contaminación de los recursos hídricos y de una extracción equitativa del sector privado; y la obligación de cumplir con los derechos al agua, mediante medidas que garanticen el acceso a las comunidades que no lo tienen (Barlow, 2012).

Ante estas obligaciones que tendrían los Estados pertenecientes a la ONU en la medida en que fueran jurídicamente vinculantes $y$ ante las apremiantes necesidades de agua potable de la población mundial, ¿podría explotarse el agua de la Antártida? En las líneas subsiguientes se tratará de dilucidar esta pregunta.

\section{El sistema del Tratado Antártico y el agua}

En octubre de 1959 tuvo lugar la Conferencia de Washington, cuyo resultado fue el Tratado Antártico (en adelante TA), que es el pilar del llamado Sistema del Tratado Antártico (en adelante STA ${ }^{7}$. Mediante este tratado se garantizaron una serie de principios para ser aplicados en la zona al sur de los $60^{\circ}$ grados de latitud sur (artículo VI TA), entre los cuales se encuentran el uso pacífico del territorio antártico (artículo I TA); la libertad de investigación científica y la cooperación internacional para ese fin (artículos II y III TA); la suspensión y no ampliación de las reclamaciones territoriales realizadas, así como la proscripción de nuevas reclamaciones territoriales (artículo IV TA), y el consenso en la toma de decisiones, que aunque no está expresamente consagrado en un artículo, se puede inferir de IX y XII TA ${ }^{8}$.

Si bien el TA no hizo referencia expresa al uso de los minerales antárticos, sí reconoció en su preámbulo que es "interés de toda la humanidad que la Antártida continúe utilizándose siempre exclusivamente para fines pacíficos y que no llegue a ser escenario

Un estudio pormenorizado de cómo se fue gestando la Conferencia de Washington y el Tratado Antártico puede consultarse, entre otros, en Díaz \& Villamizar (2014).

8 Para profundizar sobre los principios del Tratado Antártico puede consultarse, entre otros, Villamizar (2013), texto en el cual se analiza cada uno de los principios y su respectivo alcance en el contexto del Sistema del Tratado Antártico. 
u objeto de discordia internacional". Para garantizar ese interés de toda la humanidad, y en virtud de la creciente conciencia medioambiental que se empezó a dar en el mundo en general y en la Antártida en particular, poco a poco se empezaron a proferir diversos mecanismos jurídicos para regular las actividades antárticas que pudieran tener efectos nocivos en el ambiente antártico ${ }^{9}$, primero respecto de los organismos vivos ${ }^{10}$.

En cuanto a la regulación sobre los recursos minerales antárticos, hubo en su momento una posición que afirmaba la improcedencia de una regulación especial en ese sentido, debido a que ese tema era posible regularlo mediante el Derecho Consuetudinario (Kindt, 1988); sin embargo, rápidamente esta posición pasó a ser minoritaria debido a que las particularidades del STA hacían imposible el uso de analogías basadas en otros sistemas (Pineshi, 1996). En ese sentido, surgió la necesidad de regular positivamente los temas concernientes a los minerales antárticos, y en ese contexto se fue erigiendo la Convención para la Reglamentación de las Actividades sobre Recursos Minerales Antárticos, (en adelante Cramra), de Wellington en 1988, y el Protocolo al Tratado Antártico sobre Protección del Medio Ambiente de 1991, conocido como el Protocolo de Madrid en razón de la ciudad en donde se adoptó.

Cramra fue producto de una serie de negociaciones sobre minerales que se llevaron a cabo desde 1982 en el seno de la Reunión Consultiva del Tratado Antártico ${ }^{11}$ por los Estados que tenían en ese momento estatus de parte consultiva $^{12}$, pese a las reiteradas solicitudes de la ONU de una moratoria ${ }^{13}$. Cramra preveía una

9 Sobre la evolución del Sistema del Tratado Antártico puede consultarse, entre otros, Ferrada (2012), quien hace un análisis de cómo de un entorno en el cual primaban las necesidades geoestratégicas progresivamente se empezó a gestar una conciencia medioambiental que en buena medida es un reflejo de los requerimientos para un correcto funcionamiento de la gobernanza antártica.

10 Entre los que se destacan las "Medidas Convenidas para la Protección de Flora y Fauna Antártica", adoptada en Bélgica en 1964, la "Convención para la Conservación de las Focas Antárticas", adoptada en Londres en 1972, y la "Convención para la Conservación de los Recursos Marinos Antárticos", adoptada en Camberra en 1980.

11 La Reunión Consultiva del Tratado Antártico es el órgano de decisión más importante del Sistema del Tratado Antártico y está prevista en el artículo IX del TA.

12 El Tratado Antártico divide las partes en consultivas y no consultivas. Las partes consultivas están constituidas por los fundadores del TA, por derecho propio, y quienes de conformidad con el artículo IX del TA, numeral 2, demuestren "su interés en la Antártica mediante la realización en ella de investigaciones científicas importantes, como el establecimiento de una estación científica o el envío de una expedición científica". Las partes no consultivas son quienes se han adherido al TA pero no son fundadores o no han demostrado el interés científico en los términos exigidos por el artículo IX numeral 2 TA.

13 Pueden consultarse, entre otras, las siguientes resoluciones sobre ese particular: U.N. Resolution 41/88, 1988; U.N. Resolution 42/46B, 1988; 43/83, 1988. 
serie de objetivos, principios, reglas e instituciones encaminadas a regular la explotación de minerales antárticos. De hecho, expresamente se establecía que:

No se efectuarán actividades sobre recursos minerales antárticos si no es en conformidad con esta Convención, y con las medidas en vigor en virtud de ella y, en los casos de exploración y explotación, con un Esquema de Administración aprobado conforme a los Artículos 48 ó 54. (Cramra, artículo 3).

Pese a la protección ambiental prevista en Cramra, diversos actores, como Antarctic and Southern Ocean Coalition y Greenpeace, criticaban fuertemente que se permitiera la explotación de minerales y consideraban que la explotación comercial dañaría el medio ambiente antártico. En razón de esto, Australia y Francia, que son líderes en materia antártica y miembros fundadores del TA, se negaron hacerse parte de Cramra en el período previsto para ser ratificada (del 25 de noviembre de 1988 al 25 de noviembre de 1989). La actitud de estos dos Estados disuadió a otros a ratificar esta convención, y esto llevó a que no se cumpliera con el número de miembros consultivos requeridos para la entrada en vigor del Cramra, que según el artículo 62 de esta era de 16 miembros consultivos. Muchos miembros consultivos ni siquiera intentaron ratificarla, pese a criticar la decisión franco-australiana, como fue el caso de Nueva Zelanda (Davis, 1990).

Dado el fracaso que supuso la falta de ratificación de Cramra y la consecuente imposibilidad jurídica de hacer que sus provisiones fueran exigibles, el tema de los minerales quedó sin regularse hasta 1991, año en el que tuvo lugar el Protocolo de Madrid. Según el artículo 2 de dicho instrumento, "Las Partes se comprometen a la protección global del medio ambiente antártico y los ecosistemas dependientes y asociados y, mediante el presente Protocolo, designan a la Antártida como reserva natural, consagrada a la paz y a la ciencia". Además de las diferentes obligaciones que implican los postulados del artículo citado, el artículo 7 del Protocolo de Madrid zanjó de manera expresa cualquier posibilidad de explotar comercialmente los minerales antárticos mediante el siguiente tenor literal: "Cualquier actividad relacionada con los recursos minerales, salvo la investigación científica, estará prohibida".

En ese sentido y analizando solo el tenor literal del artículo 7 del Protocolo de Madrid, exclusivamente se podría hacer algo relativo al agua de la Antártida si tiene fines científicos. $\mathrm{Ni}$ siquiera se contemplan fines humanitarios, que podrían generarse como consecuencia de una gran sequía. Adicionalmente, se debe tener en cuenta el fallo de 2014 de la Corte Internacional de Justicia (CIJ, 2014), por el cual se limita la posibilidad de 
ampararse en programas científicos para realizar actividades comerciales, con lo cual aun la explotación para fines científicos queda seriamente restringida, porque con los argumentos de la CIJ no basta alegar unilateralmente que se están realizando actividades de investigación científica, como lo hacía Japón para cazar ballenas, sino que se deben cumplir estrictamente una serie de requisitos, como el análisis de los objetivos de investigación, que deben ser considerados a la hora de esgrimir que se está haciendo investigación científica $^{14}$.

Además, la institucionalidad del STA, como el Comité Científico (SCAR por sus siglas en inglés) y el Comité de Protección Ambiental (CEP por sus siglas en inglés), y los programas científicos de los Estados miembros no deberían permitir desviaciones de la finalidad científica de actividades relativas a minerales.

Así las cosas, considerando que el agua es un mineral, sería verdaderamente difícil emplear el agua antártica para paliar las crecientes necesidades de agua potable en el mundo debido a la prohibición de explotar minerales antárticos, contenida en el artículo 7 del Protocolo de Madrid. De manera que la pregunta que ha inspirado este trabajo debería ser respondida de forma negativa: con la regulación actual de minerales del STA solo se puede explotar agua para fines científicos, no para fines humanitarios ni mucho menos para fines comerciales. Sin embargo, como se verá a continuación, en el Acta Final de la XI Reunión Consultiva Especial del Tratado Antártico hay una constancia por la cual se puede interpretar que sí es posible explotar el agua antártica aunque de manera restringida.

Acta final de la XI Reunión Consultiva Especial del Tratado Antártico: ¿la llave para la explotación de agua antártica?

El cuatro de octubre de 1991 en Madrid finalizó la XI Reunión Consultiva Especial del Tratado Antártico. A dicha Reunión Consultiva Especial acudieron los entonces miembros consultivos del TA, miembros no consultivos y diversos observadores representantes de organizaciones internacionales gubernamentales $y$ no gubernamentales. El objetivo fundamental de este cónclave era que las partes consultivas adoptaran el «Protocolo al Tratado Antártico sobre Protección del Medio Ambiente» (Protocolo de Madrid) y cuatro

14 Sobre este tema, la Corte Internacional de Justicia fija un estándar de revisión para determinar qué programa de investigación puede considerarse como científico y fija diversos parámetros complementarios para ayudar a lograr ese fin, con lo cual desatiende la pretensión japonesa según la cual su programa JARPA II tenía un carácter científico (Villamizar, 2016). 
Anexos al Protocolo, que formarían parte integrante del mismo, con la posibilidad de incorporar nuevos anexos, como en efecto ha ocurrido.

Junto con la proclamación del Protocolo de Madrid, se dictó un Acta Final cuya interpretación puede dar un giro a cómo deben entenderse las prohibiciones en materia de explotación de minerales del Protocolo de Madrid. Como se vio anteriormente, en el artículo 7 del mencionado protocolo se prohíbe la explotación de minerales, dentro de los cuales por su naturaleza se incluiría el agua, sin embargo en el Acta Final del evento se hace una declaración que cambia sustancialmente la interpretación del mencionado artículo, y se hace en los siguientes términos:

La Reunión tomó nota que la explotación de hielo no era considerada una actividad relativa a los recursos minerales antárticos; se acordó, por tanto, que si la explotación de hielo llegara a resultar posible en el futuro, se entendía que serían aplicables las disposiciones del Protocolo, con excepción del artículo 7. (Acta Final XI Reunión Consultiva Especial del Tratado Antártico, par. 6).

De esta declaración surgen una serie de preguntas de difícil resolución, pero que a continuación se tratarán de resolver. Dichas preguntas son: ¿Cómo debe interpretarse entonces el artículo 7 del Protocolo de Madrid a la luz de esta declaración? ¿Si se entiende que la declaración tiene un valor jurídico por el cual da una excepción al artículo 7 del Protocolo de Madrid, la explotación de hielo puede interpretarse como explotación de agua? ¿Si jurídicamente se puede explotar el agua en forma de hielo, y fuera viable comercialmente o para fines humanitarios, qué regulaciones tendrían que cumplirse? ¿Qué efectos tendría la explotación de agua respecto de los Estados reclamantes de soberanía?

Respecto de la primera pregunta sobre la interpretación de la declaración del Acta Final frente a la prohibición de la explotación de minerales del Protocolo de Madrid, consideramos que la respuesta puede encontrarse en el numeral 2 del artículo 31 de la Convención de Viena sobre el Derecho de los Tratados de $1969^{15}$, pues en virtud de ese precepto se entiende que para la interpretación de un tratado es necesario considerar el contexto del mismo, y dentro de los elementos que componen dicho contexto, además del texto del tratado, con su preámbulo y anexos, se tiene en cuenta "todo acuerdo que se refiera al tratado y

15 El tenor literal de esa norma es: “(...) 2. Para los efectos de la interpretación de un tratado el contexto comprenderá, además del texto, incluidos su preámbulo y anexos: a) todo acuerdo que se refiera al tratado y haya sido concertado entre todas las partes con motivo de la celebración del tratado". 
haya sido concertado entre todas las partes con motivo de la celebración del tratado", y comoquiera que en el Acta Final consta el acuerdo de las partes para permitir la explotación futura de hielo, debe entenderse entonces que el artículo 7 del Protocolo de Madrid, que prohíbe la explotación de minerales antárticos salvo para fines científicos, no es aplicable para la mencionada explotación de hielo y por tanto se podría explotar el hielo antártico.

En ese orden de ideas, la segunda pregunta relativa a si la explotación de hielo puede interpretarse como explotación de agua requiere de cierta reflexión, porque si bien es evidente que el hielo es agua congelada, no habría mucho que razonar en el sentido de que si se autoriza la explotación de hielo se está intrínsecamente permitiendo explotar agua antártica, pero dado que en la Antártida hay agua dulce de ríos, corrientes interiores y lagos (Vincent \& Laybourn-Parry, 2008) podría haberse aludido directamente a la explotación de agua si se hubiera querido, porque si hay una diferenciación es por algún motivo o para que tenga un efecto razonable.

En los Informes públicos de la XI Reunión Consultiva Especial del Tratado Antártico ${ }^{16}$ no hay evidencia que demuestre el porqué de la alusión al hielo y no al agua directamente (Sistema del Tratado Antártico, 2016). En consecuencia, para resolver este intríngulis, es menester acudir de nuevo al artículo 31 de la Convención de Viena sobre el Derecho de los Tratados. Bajo ese precepto legal, la referencia a la explotación de hielo debe interpretarse de buena fe conforme al sentido corriente que haya de atribuirse a los términos del tratado en el contexto de estos y teniendo en cuenta su objeto y $\mathrm{fin}^{17}$.

Portanto, asumiendounainterpretación de buena fe que considera el fin del Protocolo de Madrid, que es la protección ambiental antártica, estimamos que se podría explotar el hielo antártico de conformidad con lo establecido en el pacto del Acta Final, y una vez que este se derrita, es decir se convertirá en agua, podrá utilizarse para los fines pretendidos por el

16 La IX Reunión Consultiva Especial del Tratado Antártico tuvo lugar en cuatro sesiones. La primera fue en Viña del Mar, Chile, del 19 de noviembre al 6 de diciembre de 1990. La segunda del 22 al 30 de abril de 1991 en Madrid. La tercera del 17 al 22 de junio de 1991, y la cuarta del 3 al 4 de octubre de 1991 también en Madrid.

17 El propio Protocolo de Madrid, en su artículo 2, establece como objetivo del mismo: "Las Partes se comprometen a la protección global del medio ambiente antártico y los ecosistemas dependientes y asociados y, mediante el presente Protocolo, designan a la Antártida como reserva natural, consagrada a la paz y a la ciencia". 
explotador del recurso, pero las aguas antárticas contenidas en ríos, lagos, corrientes internas y todas aquellas que no provengan de hielo, deberían estar bajo el cobijo de la prohibición de la explotación de minerales del artículo 7 del Protocolo de Madrid.

Es decir, para ser más claros, respecto de las aguas antárticas que no sean hielo debería operar la prohibición de explotación de minerales, esto es para aguas subterráneas, ríos, lagos, etc. Según el Acta Final sub examine, solo es posible explotar hielo antártico, que después de un proceso de aclimatación ese hielo se convertiría en agua.

Asumiendo, entonces, que es lícito explotar el hielo antártico de conformidad con el Acta Final, la tercera pregunta planteada versa sobre qué regulaciones deben cumplirse para esos efectos. La respuesta a este interrogante es que deben cumplirse todas las disposiciones establecidas en el Protocolo de Madrid y sus anexos, como por ejemplo los principios de planificación para evitar daños al ambiente antártico, de información suficiente, evaluación ambiental previa, juicio razonado sobre el impacto ambiental, entre otros ${ }^{18}$. No habría ninguna excepción para dejar de cumplir con las regulaciones del STA para realizar actividades en la Antártida.
En cuanto a la respuesta a la cuarta pregunta planteada, sobre los efectos que tendría la explotación de agua respecto de los Estados reclamantes de soberanía, no es fácil de responder, pero hay elementos que permiten intuir lo que probablemente ocurriría.

Cuando se revisa la historia de la Antártida, se puede fácilmente dilucidar que el éxito fundamental del STA ha sido el hecho de evitar un conflicto por los recursos antárticos (Martin, 2013; Howkins, 2016). En el caso de que se hiciera una explotación de hielo lo más probable es que los diferentes interesados harían valer sus pretensiones territoriales, y temas como el reparto de beneficios serían tan críticos que muy probablemente darían lugar a conflictos de, al menos, envergadura considerable.

Dado que uno de los objetivos fundamentales del Tratado Antártico y del sistema que de él deriva es el uso de la Antártida exclusivamente para fines pacíficos y la continuación de la armonía internacional en ese continente, pensar en una explotación comercial del hielo antártico para convertirlo en agua es muy difícil, aun si se cumplen todos los requisitos de la normatividad del STA y la viabilidad tecnológica y financiera lo permiten. En ese sentido, la explotación del hielo antártico debería darse en la práctica

18 Para una referencia pormenorizada de los principios ambientales antárticos contenidos en el Protocolo de Madrid puede consultarse, entre otros, Villamizar (2015). 
para asuntos muy restrictivos, como lo podría ser una crisis humanitaria puntual o circunstancias de extrema gravedad que impidan que los Estados reclamantes de soberanía en la Antártida y otros interesados sientan que sus derechos reales o potenciales se vean afectados.

En ese orden de ideas, se podría afirmar que si bien jurídicamente es viable la explotación del hielo antártico como consecuencia del acuerdo plasmado en el Acta Final de la XI Reunión Consultiva Especial del Tratado Antártico, en la práctica los efectos en las configuraciones de poder limitarían de manera sustancial esta práctica, porque de darse de forma irrestricta, la estabilidad brindada por el STA se vería seriamente afectada y la escalada en los conflictos podría ser de dimensiones considerables, y por qué no planetarias, porque los intereses en la Antártida cada día son mayores y más actores no tradicionales se involucran de manera seria, como es el caso de China, India y Corea del Sur, solo por mencionar algunos de los más notables en los tiempos recientes (Romero, 2015).

\section{Conclusión}

En las líneas iniciales de este trabajo se planteaba la pregunta sobre si podría utilizarse el agua dulce de la Antártida para atender la creciente necesidad de agua del planeta. Esta pregunta tan amplia debía considerar la actual, y probablemente creciente, normatividad jurídica internacional que pretende obligar a los Estados a procurar el acceso al agua potable a sus ciudadanos. A su vez, en la pregunta subyace que las fuentes acuíferas cada vez son más escasas y las necesidades por estas aumentan día a día en forma exponencial. Así las cosas, la Antártida debería ser una posibilidad de alivio para satisfacer en alguna medida la escasez de agua, pero el STA, concretamente el Protocolo de Madrid, en principio cierra cualquier posibilidad de explotar el agua antártica, pues cualquier actividad con minerales está prohibida salvo para fines diferentes a los científicos.

Sin embargo, pese a la prohibición de actividades que involucren minerales, el Acta Final de la XI Reunión Consultiva Especial del Tratado Antártico abre, en principio, una posibilidad para obtener agua antártica en la medida en que los miembros consultivos del TA y otras partes interesadas dejaron expresa constancia de un pacto por el cual la prohibición de explotación de minerales no sería aplicable a la explotación de hielo, de manera que por esta vía se podría explotar agua antártica y la respuesta al interrogante inicial, por lo menos teóricamente, sería afirmativa, es decir que sí se puede utilizar el agua antártica para satisfacer las necesidades mundiales siempre que la explotación sea en forma de hielo, y en ese sentido la hipótesis se confirmaría en buena medida. 
Esta posibilidad teórica de explotar legalmente el hielo antártico, y con este conseguir agua, debería realizarse de conformidad con las regulaciones del Protocolo de Madrid en materia de protección ambiental antártica, pero en todo caso se ve seriamente comprometida por los diversos intereses presentes en la Antártida, más allá de si es posible realizar dicha explotación desde un punto de vista financiero y tecnológico. Es arduo pensar que los diferentes Estados del mundo, y no solo aquellos con intereses en la Antártida, permitan la explotación de hielo antártico sin mayores dificultades o solo permitiendo cumplir los requisitos establecidos en el STA.

Además de las pasiones que puede generar la explotación de hielo antártico en los países con reclamaciones territoriales en ese continente, los diferentes actores no gubernamentales y Estados con intereses en la Antártida también harían sentir sus respectivas posiciones con respecto a esa explotación, con lo cual los fines pacíficos en el uso del territorio antártico se verían seriamente afectados y es probable que el STA sucumbiría ante tanta presión. En ese contexto cabe preguntarse si antes de ejercer presiones excesivas al STA podría alcanzarse un consenso mundial para lograr la explotación de hielo antártico para casos extremos, como crisis humanitarias de grandes proporciones.
Creemos que lograr ese consenso es tema bastante difícil, porque por muy buena voluntad que haya por parte de todos los involucrados, dado el frágil equilibrio de los ecosistemas antárticos, la posibilidad de alterarlos con una explotación masiva es muy alta, aunque dicha explotación no sea con fines comerciales. Las preguntas que surgen entonces son: ¿Qué hacer mientras se llega a ese consenso? ¿Será que el consenso llegará debido a los múltiples conflictos que se anuncian por causa del acceso al agua, o precisamente por esos conflictos no se arribará a un consenso? Estas y otras preguntas solo el tiempo podrá resolverlas, pero por lo pronto, aunque jurídica, financiera y tecnológicamente sea posible explotar el hielo antártico para obtener agua, las condiciones políticas no parecen ser las mejores para esos efectos, o por lo menos para una explotación relativamente masiva.

\section{REFERENCIAS}

Asamblea General de la Organización de Naciones Unidas. (2010). Resolución 64/292 de 28 de julio de 2010. Recuperado de: http://www. un.org/spanish/waterforlifedecade/ human_right_to_water.shtml.

Barlow, M. (2012). "Foreword". En Farhana Sultana \& Alex Loftus (Eds.), The Right to Water. Politics, Governance and Social Struggles. New York: Earthscan. 
Bertazzo, S. (2015). La tutela del acceso al agua potable en el Derecho Internacional. Revista de Derecho Universidad Católica del Norte, 22 (2), 55-92.

Comité de Derechos Económicos, Sociales y Culturales de la Organización de Naciones Unidas (2010). Observación General № 15 sobre el derecho al agua. Recuperado de: http://www.un.org/ga/search/ view_doc.asp?symbol=A/ $\mathrm{RES} / 6 \overline{4} / 292 \&$ Lang $=\mathrm{S}$.

Davis, A. (1990). Protecting Antarctica: Will a Minerals Agreement Guard the Door or Open the

Door to Commercial Exploitation? The George Washington journal of international law and economics, 23 (3). 733-768.

De Albuquerque, C. (2012). On the right track. Good practices in realising the rights to water and sanitation. Recuperado de: http:// www.ohchr.org/Documents/Issues/ Water/BookonGoodPractices en.pdf.

Díaz, I. \& Villamizar, F. (2014). Uso pacífico de la Antártida como norma de ius cogens. Revista Magallania, 42 (1), 17-31.

Diplomatie, Affaires Stratégiques et Relations Internationales (2014, Décembre - Janvier). L'eau, d'aujourd'hui... à demain, Les Grands Dossiers No. 18 Atlas Géostratégique, p. 14.
Ferrada, L. (2012). Evolución del Sistema del Tratado Antártico: desde su génesis geoestratégica a sus preocupaciones ambientalistas. Revista de Derecho Universidad San Sebastián (Chile), 18, 131-151.

Ferrigno, J., Cook, A., Mathie, A., Williams, R., Swithinbank, C., Foley, K., .... Sievers, J. (2009). CoastalChange and Glaciological Map of the Palmer Land Area, Antarctica: 1947-2009. USA, US Geological Survey, British Antarctica Survey, Scott Polar Research Institute, U.S. Geological Survey Geologic Investigations Series.

Harris, S. (2014, 18 de septiembre). Water Wars. Forget the Islamic State. The new conflicts of the future could be sparked by climate change. Foreign Policy. Recuperado de: http://foreignpolicy.com/2014/ 09/18/water-wars/.

Howkins, A. (2016). The Polar Regions. An environmental History. Cambridge, USA: Polity Press.

International Court of Justice. (2014). Judgment Whaling in Antarctic Australia v. Japan, New Zealand intervening, 31 march 2014.

Kindt, J. (1988). A Regime for IceCovered Areas: The Antarctic and Issues Involving Resource Exploration and the Environmental. En C. Joyner \& S. Chopra (Eds.), The Antarctic Legal Regime (pp. 187-218). Dordrecht, Netherlands, Martinus - Nijhoff Publishers. 
Martin, S. (2013). A History of Antarctica. Australia: Rosenberg Publishing.

Martínez, A. \& Defelipe, Ó. (2013). Derecho humano al agua y control de convencionalidad. Revista Derecho Pontificia Universidad Católica del Perú, 70, pp. 105120. Recuperado de: http:// revistas.pucp.edu.pe/index.php/ derechopucp/article/view/6746.

Motta, R. (2010). El derecho humano al agua potable: entre un reconocimiento popular y jurisprudencial. En Revista Misión Jurídica. Revista de Derecho y Ciencias Sociales, 3, pp. 257272. Recuperado de: http:// www.unicolmayor.edu.co/revis tajuridica/pdf/terceraedicion/el derechohumanoalaguapotable.pdf.

Office of the Director of National Intelligence. (2014). The National Intelligence Strategy of the United States of America 2014. USA.

Pineschi, L. (1996). The Antarctic Treaty System and General Rules of International Environmental Law. F. Francioni \& T. Scovazzi (Eds.) Droit International de l'Antartique (pp. 187-246), Milano: Guffré.

Pinto, M. et al. (2008). El derecho humano al agua: particularidades de su reconocimiento, evolución y ejercicio. Buenos Aires: Abeledo Perrot.

Romero, S. (2015, 29 de diciembre). Countries Rush for Upper Hand in Antarctica. New York Times. Recuperado dehttp://www.nytimes. com/interactive/2015/12/29/ worldcountries-rush-for-upperhand-antarctica.html?action $=$ click \&contentCollection $=$ Americas \& module $=$ RelatedCoverage $\&$ re gion $=$ Marginalia \&pgtype $=$ article .

Shaw, M. (2011). International Law. Sixth edition, $5^{\text {th }}$ printing, New York: Cambridge University Press.

Sistema del Tratado Antártico. (2016). Informes sobre la XI Reunión Consultiva Especial del Tratado Antártico. Recuperado http:// www.ats.aq/devAS/info_finalrep. aspx?lang $=$ s\&menu $=5$

Sistema del Tratado Antártico. Acta Final de la XI Reunión Consultiva Especial del Tratado Antártico. Recuperado de: http://www.ats. aq/documents/keydocs/vol_1/ vol1_3_AT_Final_Act_Eleventh_ SATCM_e.pdf

United Nations Economic Commission for Europe (Unece). (2013). The Equitable Access Score-card: Supporting policy processes to achieve the human right to water and sanitation. Recuperado de: http://www.unece.org/fileadmin/ DAM/env/water/publications/ PWH_equitable_access/1324456 ECE_MP_WP_8_Web_Interactif_ ENG.pdf.

Villamizar, F. (2013). Antarctic Treaty and Antarctic Territory Protection Mechanisms. Revista Chilena de Derecho, 40 (2), 461-488. 
Villamizar, F. (2015). El tratamiento doctrinal de la bioprospección como desafío al Derecho Antártico. En Manuel Núñez (Ed.), La internalización del Derecho Público (pp. 259-273). Santiago de Chile: Thompson Reuters - La Ley. Villamizar, F. (2016). Comentarios a la sentencia del caso "Whaling in the Antarctic", Australia v. Japón (Nueva Zelanda inter.). Anuario Colombiano de Derecho Internacional, 9, 81-112.

Vincent, W. \& Laybourn-Parry, J. (2008). Polar lakes and rivers: limnology of Arctic and Antarctic aquatic ecosystems. Oxford: Oxford University Press.

Winkler, I. (2012). The Human Right to Water. Significance, Legal Status and Implications for Water Allocation. United Kingdom: Hart Publishing Ltd.

Xinhuanet. (2014, 09 de diciembre). China envía más agua potable a Maldivas. Xinhuanet. Recuperado de: http://spanish.xinhuanet.com/ photo/2014-12/09/c_133841213. htm. 